\title{
Diffusion tractography for awake craniotomy: accuracy and factors affecting specificity
}

\author{
Natalie L. Voets ${ }^{1}\left[\right.$ Pieter Pretorius ${ }^{3} \cdot$ Martin D. Birch ${ }^{4} \cdot$ Vasileios Apostolopoulos $^{1} \cdot$ Richard Stacey $^{1} \cdot$ Puneet Plaha $^{1,2}$
}

Received: 29 April 2021 / Accepted: 22 June 2021 / Published online: 1 July 2021

(c) The Author(s) 2021

\begin{abstract}
Introduction Despite evidence of correspondence with intraoperative stimulation, there remains limited data on MRI diffusion tractography (DT)'s sensitivity to predict morbidity after neurosurgical oncology treatment. Our aims were: (1) evaluate DT against subcortical stimulation mapping and performance changes during and after awake neurosurgery; (2) evaluate utility of early post-operative DT to predict recovery from post-surgical deficits.

Methods We retrospectively reviewed our first 100 awake neurosurgery procedures using DT- neuronavigation. Intra-operative stimulation and performance outcomes were assessed to classify DT predictions for sensitivity and specificity calculations. Post-operative DT data, available in 51 patients, were inspected for tract damage.

Results 91 adult brain tumor patients (mean 49.2 years, 43 women) underwent 100 awake surgeries with subcortical stimulation between 2014 and 2019. Sensitivity and specificity of pre-operative DT predictions were $92.2 \%$ and $69.2 \%$, varying among tracts. Post-operative deficits occurred after 41 procedures (39\%), but were prolonged ( $>3$ months) in only 4 patients (4\%). Post-operative DT in general confirmed surgical preservation of tracts. Post-operative DT anticipated complete recovery in a patient with supplementary motor area syndrome, and indicated infarct-related damage to corticospinal fibers associated with delayed, partial recovery in a second patient.

Conclusions Pre-operative DT provided very accurate predictions of the spatial location of tracts in relation to a tumor. As expected, however, the presence of a tract did not inform its functional status, resulting in variable DT specificity among individual tracts. While prolonged deficits were rare, DT in the immediate post-operative period offered additional potential to monitor neurological deficits and anticipate recovery potential.
\end{abstract}

Keywords Glioma · Diffusion tractography $\cdot$ DTI $\cdot$ Awake surgery $\cdot$ Stimulation

Puneet Plaha

puneet.plaha@nds.ox.ac.uk

1 Department of Neurosurgery, Oxford University Hospital NHS Foundation Trust, John Radcliffe Hospital, West Wing, L3, Oxford, Oxfordshire OX3 9DU, UK

2 Nuffield Department of Surgery, University of Oxford, Oxford, Oxfordshire, UK

3 Department of Neuroradiology, Oxford University Hospital NHS Foundation Trust, John Radcliffe Hospital, Oxford, Oxfordshire, UK

4 Nuffield Department of Anaesthesia, Oxford University Hospital NHS Foundation Trust, John Radcliffe Hospital, Oxford, Oxfordshire, UK

\section{Introduction}

Surgery remains the first-line treatment for intrinsic brain tumors. Surgery has undergone increasing refinement with adjuncts that help maximize tumor resection and reduce morbidity. Evidence for the added value of emerging intraoperative approaches, however, remains low [1].

Neuronavigation incorporating diffusion tractography (DT) has received particular attention for its potential to facilitate glioma surgery [2,3]. Maximal resection optimizes overall and progression-free survival in low-grade $[4,5]$ and high-grade gliomas [6]. However, the often-eloquent location of gliomas [7] creates challenges in deciding how to maximize resection without causing neurological deficits. Among the primary factors limiting resection is the location of eloquent subcortical white matter pathways [8]. Electrical stimulation mapping and neurophysiological monitoring 
studies highlight the need to spare deep fiber tracts to maximize quality of life [9]. Pre-surgical indicators of the location of functionally relevant tracts, therefore, have great potential to assist with surgical decision-making.

MRI-based diffusion tractography (DT) is the only technique available to estimate fiber tracts in vivo. Although an indirect technique [10], the topography of tracts estimated from DT shows high correspondence with neuroanatomical dissections [11]. When applied in brain tumor patients, pre-operative DT can depict varied effects (including displacement or infiltration) of space occupying lesions on fiber tracts in individual patients $[12,13]$. These applications motivate clinical interest in pre-operative DT to inform safe surgical approaches [14], anticipate the likely extent of resection [15-17] and plan the need and focus for electrical stimulation $[12,18]$.

Evaluations against intra-operative electrical stimulation highlight that factors including tumor-associated edema and brain shift [19] reduce the accuracy of pre-operative DT. Nevertheless, the predictive value of DT for specific fiber tracts is generally high [20] when allowing a margin of 5-7 mm, consistent with the expected electrical current spread $[18,21]$. Of course, detecting the presence of tracts does not prove these are essential to function, driving the need for intra-operative tract monitoring. However, awake surgery is not feasible in all patients, and not infallible [22]. Consequently, there is growing interest in the potential of DT not only to anticipate intra-operative findings, but-crucially-to predict post-operative functional outcomes and recovery [23-26].

We analyzed findings in 100 patients at our institution who underwent awake surgery for intrinsic brain tumors using DT neuronavigation combined with subcortical stimulation. Our aims were to:

1. Confirm DT sensitivity, specificity and accuracy for 5 primary fiber tracts, evaluated not only against intraoperative stimulation but also against behavioral change during/after surgery.

2. Identify factors contributing to discrepancies between pre-surgically DT predicted tract locations and intraoperative stimulation.

3. Explore if post-operative indications of fiber tract damage inform recovery potential.

\section{Methods}

\section{Patients}

We reviewed all adults undergoing awake surgery for intrinsic brain tumors at our institution between May 2014 and 31 May 2019. Patients were selected for awake surgery if intraoperative assessment of speech, sensorimotor or visual functions was felt to be important to achieve maximal resection without morbidity based on the location of the tumor. The first 100 surgeries using intra-operative subcortical stimulation were analyzed. Patients were reviewed at a multidisciplinary meeting and dedicated surgical neurooncology clinic to assess performance status and candidacy for awake neurosurgery.

\section{MRI}

Patients underwent MRI approximately $24 \mathrm{~h}$ before surgery on a 1.5 T Philips Achieva. Scans included volumetric preand post-Gadolinium T1-weighted scan, axial T2-weighted FLAIR and diffusion (b-value: $1200 \mathrm{~s} / \mathrm{mm}^{2}, 32$ directions, $2.5 \mathrm{~mm}^{3}$ resolution). 98 patients underwent post-operative MRI approximately $24 \mathrm{~h}$ after surgery, including post-operative DT in 51 patients. Two patients had post-operative CT. Post-operative MRIs were evaluated independently by a consultant neuroradiologist to determine extent of resection.

\section{Fiber reconstructions}

Diffusion data were analyzed using commercial software (Brainlab iPlan ${ }^{\circledR}$ (Munich, Germany) or Medtronic StealthStation ${ }^{\circledR}$ S7 (Louisville, USA) using standard processes. Tracts were selected based on tumor location and included the arcuate/superior longitudinal fasciculus (SLF), inferior fronto-occipital fasciculus (IFOF), corticospinal tract (CST), optic radiations (OR) and inferior longitudinal fasciculus (ILF). Tractography plans were exported to the neuronavigation system (see Online Resource).

\section{Awake neurosurgery protocol}

Patients were operated according to a Monitored Anesthesia Care (MAC) or Conscious sedation protocol (Online Resource). Subcortical stimulation was performed when approaching tumor margins, or where DT indicated proximal fibers. Stimulation started around 6-8 mA, descending to a lower voltage on approaching tracts. Surgical resection alternating with stimulation proceeded until functional margins were encountered or planned tumor resection was achieved. Subcortical stimulation was not always reliable/ possible due to variable compliance levels or clinical events (e.g. seizures). In some cases, resection continued without stimulation, provided behavioral monitoring remained possible; in other words, patients maintained responses to test stimuli/instructions but did not comply sufficiently for stimulation and/or further stimulation was contraindicated by intra-operative focal seizures. Performance deterioration in specific tract-related functions of sudden onset, unrelated to seizures, pain, bleeding or dynamic brain traction was 
recorded as behavioral change without clear stimulationinduced response.

\section{Intra-operative assessments}

Tract functionality was assessed using standard tests [27, 28], including naming and word repetition (arcuate, SLF), Pyramids and Palm Trees semantic association test (IFOF), naming objects in opposing visual quadrants (ORs), visual naming and reading (ILF). CST proximity was assessed through involuntary movements/sensations, and loss in muscle power/range. The location of stimulation sites was visualized on the neuronavigation system and recorded.

\section{Peri-operative performance}

Immediate post-operative neurological outcome was determined over $24-48 \mathrm{~h}$ of in-patient observation. Neurological status was determined using the World Health Organization (WHO) performance status, supplemented as appropriate with neuropsychological tests probing language function (fluency, repetition, naming, reading, writing). Muscle power was measured using the Medical Research Council 5-point muscle scale. After discharge, patients returned for out-patient review within 2 weeks of surgery. Clinical reviews were 3 monthly for 'low-grade glioma' and bimonthly for 'high-grade glioma' patients until the start of adjuvant treatment.

\section{DT analyses}

A True positive (TP) prediction was defined as a tract identified by DT and confirmed by intra-operative stimulation/ behavioral change. DT findings were classified True negative (TN) if no tract was indicated near the tumor, nor found with stimulation. A False positive (FP) was defined as a tract identified by DT but not identified intra-operatively. Predictions were False Negative (FN) if no tract was located by DT but was found through stimulation. See Online Resource Fig. S1. Sensitivity was calculated as (TP)/(TP + FN); specificity as $(\mathrm{TN}) /(\mathrm{TN}+\mathrm{FP})$ and accuracy as $(\mathrm{TN}+\mathrm{TP}) /$ $(\mathrm{TN}+\mathrm{TP}+\mathrm{FN}+\mathrm{FP})$. Tracts were excluded from analysis if there was adjacent residual tumor on post-operative MRI or indications of atypicality (left-handedness or prior surgery combined with absence of expected symptoms intra-/ post-operatively).

\section{Post-operative DT}

Fiber tracts were separately reconstructed from the postoperative scans and visually compared to pre-operative trajectories for gross evidence of surgical tract damage.
We expected deficits would persist given evidence of tract injury, or be transient if tracts remained intact.

\section{Statistical analyses}

Statistical analyses were performed using SPSS v25. Group comparisons consisted of independent samples t-tests. Chisquare tests compared the frequency of specific DT predictions (i.e. True Positive, etc.) by performance outcome, and the contribution of histopathological diagnosis on DT predictions. Significance was set at $\mathrm{p}<0.05$.

\section{Results}

\section{Demographics}

Table 1 details clinical and histo-molecular data for 100 awake procedures in 91 patients (48 men, mean age: 49.2 years, range: $19-74$ years). Resection was radiologically gross total (100\% tumor resection, 40 patients), near total (90-99\% resection, 28 patients) or subtotal $(<90 \%, 32$ patients).

\section{Fiber tract evaluations}

143 tracts were evaluated during 100 surgeries (Fig. 1). 51 were CST, 29 arcuate/SLF, 37 IFOF, 19 optic radiations and 7 ILF.

\section{DT true positive}

95 tracts were identified with subcortical stimulation. Another 12 were identified through intra-operative performance decline $(n=5)$, post-operative deficits $(n=4)$ or both $(\mathrm{n}=3)$, each involving language tracts (4 Arcuate, 7 IFOF, 1 ILF). Together, 107 DT predictions were classified 'TP'.

\section{DT true negative}

Stimulation was performed to verify 9 tracts which DT indicated should be remote from the surgical margins. For all 9, no stimulation-related behavioral changes occurred, confirming ' $\mathrm{TN}$ ' predictions.

\section{DT false negatives}

One tract was not accurately predicted by DT. This patient showed malignant transformation of a known diffuse left temporo-fronto-insula astrocytoma. DT indicated white matter within the tumor. However, the IFOF was not reliably reconstructed due to confounding pathological signal in the temporo-parietal white matter (Fig. 2). 
Table 1 Clinical and demographic data for 91 patients constituting our first 100 awake surgeries

\begin{tabular}{|c|c|}
\hline (Parameter/Variable) & $\begin{array}{l}\text { Value/ } \\
\text { Number } \\
(\%)\end{array}$ \\
\hline \multicolumn{2}{|l|}{ Age (years), $n=91$} \\
\hline Mean & 49.2 \\
\hline Range & $19-74$ \\
\hline \multicolumn{2}{|l|}{$\operatorname{Sex}(n=91)$} \\
\hline Male & 48 \\
\hline Female & 43 \\
\hline \multicolumn{2}{|l|}{ Symptoms at presentation $(\mathrm{n}=91)$} \\
\hline Seizure/collapse & 49 \\
\hline Headache/nausea/confusion & 6 \\
\hline Neurology & 25 \\
\hline Incidental finding & 2 \\
\hline Surveillance & 8 \\
\hline Unknown & 1 \\
\hline \multicolumn{2}{|l|}{ Hemisphere of surgery $(n=100)$} \\
\hline Left & 66 \\
\hline Right & 34 \\
\hline \multicolumn{2}{|l|}{ Type of surgery $(n=100)$} \\
\hline First operation & 79 \\
\hline First awake surgery (re-do operation) & 12 \\
\hline Repeat awake surgery & 9 \\
\hline \multicolumn{2}{|l|}{ Radiological tumor resection $(n=100)$} \\
\hline Gross total & 40 \\
\hline Near total (>90\% resection) & 28 \\
\hline Subtotal $(<90 \%)$ & 32 \\
\hline \multicolumn{2}{|l|}{ WHO tumor grade $(n=100)$} \\
\hline$I I$ & 20 \\
\hline Oligodendroglioma & 11 \\
\hline Astrocytoma & 9 \\
\hline III & 27 \\
\hline Oligodendroglioma & 8 \\
\hline Astrocytoma & 18 \\
\hline Clear cell ependymoma & 1 \\
\hline$I V$ & 51 \\
\hline Metastasis & 2 \\
\hline \multicolumn{2}{|l|}{ IDH status } \\
\hline IDH+ & 43 \\
\hline IDH- & 43 \\
\hline IDH unavailable & 5 \\
\hline
\end{tabular}

IDH-mutated tumors (denoted as "IDH+") included the common IDH1R132H mutation as well as rarer IDH1 and IDH2 variants WHO World Health Organization, IDH Isocitrate dehydrogenase

Intra-operative stimulation induced semantic paraphasias and naming errors, indicating eloquent IFOF within the tumor. The patient experienced mild, transient post-operative language deficits. This result was therefore a 'FN'.

\section{DT false positives}

Four stimulation-silent but DT-predicted tracts (2 optic, 1 ILF, 1 CST, 3 patients) were not explained by identified clinical factors and classified 'FP' predictions. None experienced deficits.

\section{Exclusions}

Stimulation induced seizures in two patients. Both recovered but had prolonged post-ictal states, limiting further stimulation mapping. One further patient developed an intraoperative supplementary motor area syndrome, precluding evaluation of the DT-predicted CST.

19 additional DT-predicted tracts were not identified intra-operatively. Post-operative MRI revealed residual tumor adjacent to 11/19. For 6 others, the absence of stimulation-induced errors indicated atypical functional organization. Three 'silent' tracts (1 IFOF, 1 ILF, 1 arcuate) occurred in two left-handed patients operated for a newly diagnosed left hemisphere glioma. Neither patient had language symptoms before, during or after surgery, indicating right hemisphere dominance for language. The remaining three stimulation-silent tracts ( 1 arcuate, $1 \mathrm{CST}$ and $1 \mathrm{IFOF}$ ) occurred in three patients re-operated for tumor recurrence at $8,3.7$ and 3.4 years after initial surgery following full $(\mathrm{n}=2)$ or partial $(n=1)$ behavioral recovery. Finally, substantial brain shift occurred in two glioblastomas. The pre-operative fiber tract locations (optic radiations in both) displayed on neuronavigation and the intra-operative location of stimulation visualized through the microscope became misaligned during surgery. The stimulation sites remained remote to predicted tracts, and neither patient experienced deficits. Since the DT predictions could not be conclusively verified these 22 tracts were omitted from sensitivity/specificity analyses.

\section{Tumor grade}

The frequency of DT tract predictions (TP, etc.) did not differ by tumor grade $(\chi(6)=4.3, \mathrm{p}=0.64)$, or between 'low' versus 'high' grade tumors $(\chi(3)=4.25, \mathrm{p}=0.11)$.

\section{Post-operative deficits}

Notwithstanding minor wound leaks/infections $(\mathrm{n}=4)$, surgical complication rates were low; 41/100 awake surgeries $(41 \%)$ resulted in a post-operative performance decline, relating to 52 fiber tracts (Online Resource Fig. S2). Most declines were transient. Nine patients had persisting deficits (lasting $>3$ months) but not all resulted from surgery: four 


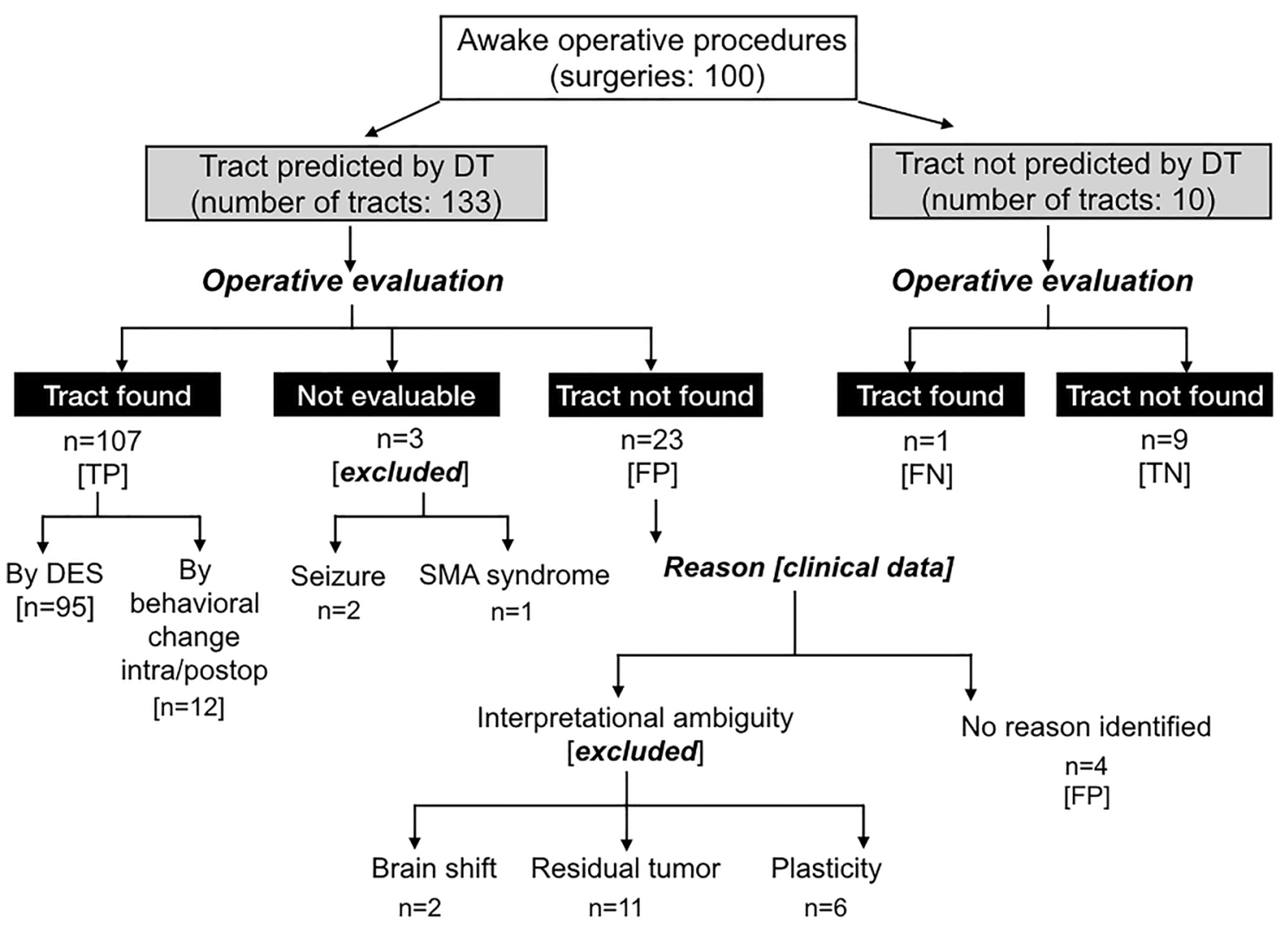

Fig. 1 Pre-operative fiber tract predictions evaluated against intraoperative subcortical stimulation and post-operative outcomes. Diffusion tractography (DT) predicted fiber locations evaluated against intra-operative direct electrical stimulation (DES) and then refined by

arose from tumor progression and one patient deteriorated upon starting radiotherapy. Therefore, definite prolonged deficits occurred after four surgeries (4\%), each involving sensorimotor functions. Tumor histological grade did not differ between patients with or without post-operative deficits $(\mathrm{t}=1.63, \mathrm{p}=0.11)$.

\section{DT sensitivity, specificity, accuracy}

107 tracts were classified TP, 1 as FN, 9 TN, and 4 FP (Fig. 2), yielding sensitivity of $92.2 \%$, specificity of $69.2 \%$ and accuracy $95.9 \%$. For values per fiber tract, see Table 2.

\section{Post-operative tract evaluations}

Post-operative DT was available in 51 patients, including 20 of those who experienced post-surgical performance declines. In 14/20 (70\%), deficits recovered rapidly. Three patients had early tumor progression contributing to neurological decline. The remaining three patients were among the four reported above with de novo deficits persisting beyond 3 months: one experienced sensory symptoms clinical data including performance outcomes and resection extent. $T P$ true positive, $F N$ false negative, $T N$ true negative, $F P$ false positive

without loss of mobility, one had persisting motor SMA syndrome, and one had residual lower limb weakness. Post-operative DT showed intact CST fibers in the patients with sensory symptoms and motor SMA syndrome. In contrast, the patient with persisting limb weakness had a small infarct involving the corona radiata, causing a postoperatively asymmetric, ipsilaterally reduced CST (Fig. 3).

\section{Discussion}

We confirmed pre-operative DT as a reliable tool for neuronavigation in neuro-oncology surgery. Addressing our first study aim, across 100 awake surgeries, DT offered $92.2 \%$ sensitivity and $95.9 \%$ accuracy to localize tracts. Secondly, lower tract-average specificity $(69.2 \%)$ reflected inherent differences between localizing fibers versus establishing their functionality. DT predicted the location of some tracts not evaluable through stimulation. Therefore, stimulation offers an important but incomplete validation of DT and maximal benefits may arise from using 


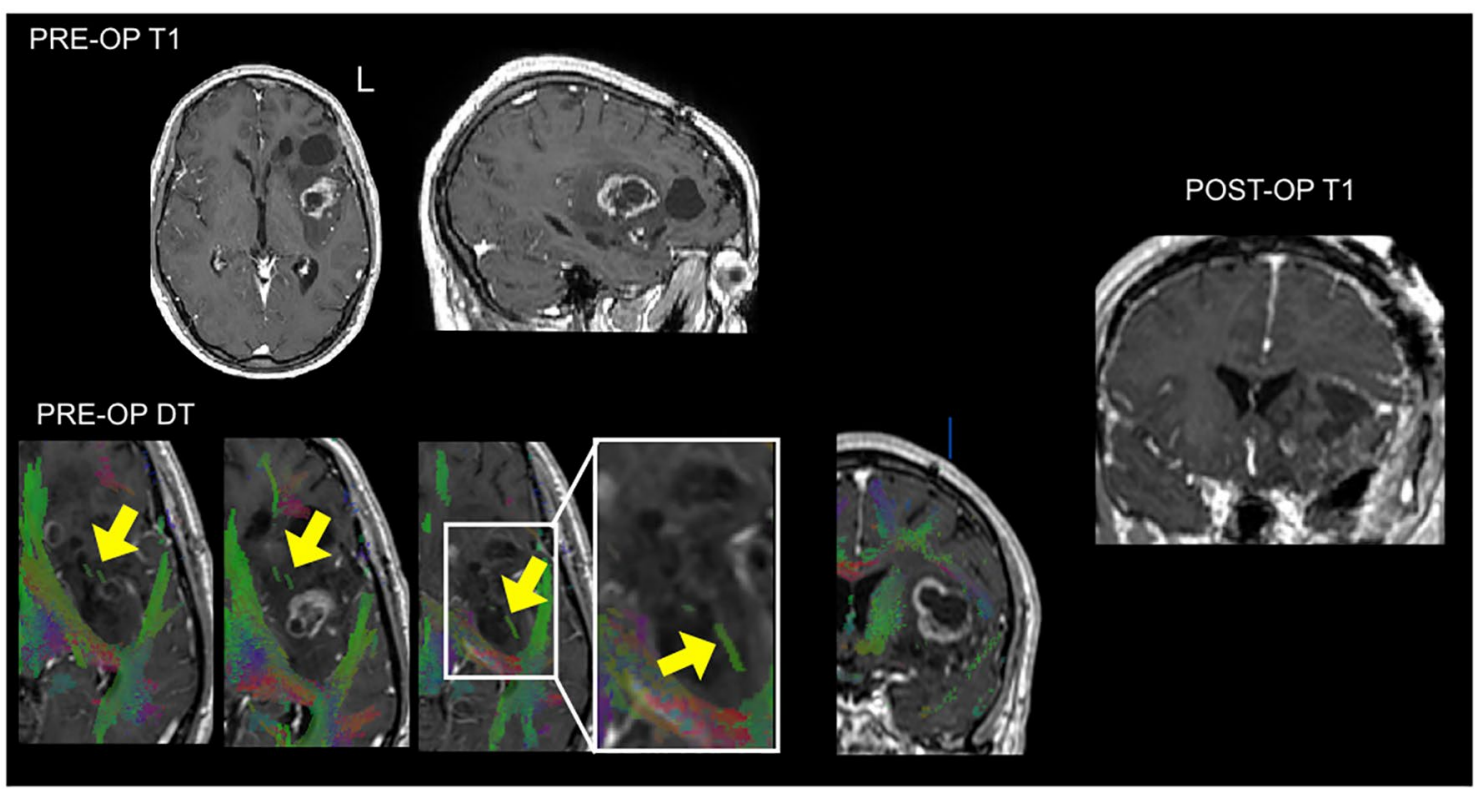

Fig. 2 Case with discordance between pre-operative DT and intraoperative stimulation. A 41-year-old right handed man on imaging surveillance for a known IDH mutated astrocytoma. Although an island of white matter and sparse 'tracts' were visible within this heterogeneous transforming lesion, pre-operative diffusion tractography (DT) did not convincingly identify the inferior fronto-occipital fasciculus running through the tumor, even at a reduced fractional anisotropy tracking threshold (0.17). However, subcortical stimulation during awake surgery elicited semantic paraphasias and naming errors at this location, indicating an eloquent IFOF. The patient experienced transient slight language deterioration affecting naming and repetition
Table 2 Sensitivity and specificity of DT predictions according to fiber tract

\begin{tabular}{lrllllllll}
\hline Tract & TP & TN & FP & FN & Exclusions & Sensitivity (\%) & Specificity & Accuracy (\%) & $\begin{array}{l}\text { EARLY } \\
\text { DEFICIT }^{\mathrm{a}}\end{array}$ \\
\hline CST $(\mathrm{n}=51)$ & 32 & 6 & 1 & 0 & 11 & 84.2 & $85.7 \%$ & 97.4 & $\mathrm{~N}=20$ \\
Arcuate $(\mathbf{n}=\mathbf{2 9})$ & 24 & 2 & 0 & 0 & 4 & 92.3 & $100 \%$ & 100 & $\mathrm{~N}=13$ \\
IFOF $(\mathbf{n = 3 7})$ & 33 & 1 & 0 & 1 & 2 & 97.1 & $100 \%$ & 97.1 & $\mathrm{~N}=13$ \\
OR $(\mathbf{n = 1 9})$ & 13 & 0 & 2 & 0 & 4 & 100 & N/A* & 86.7 & $\mathrm{~N}=4$ \\
ILF $(\mathbf{n}=7)$ & 5 & 0 & 1 & 0 & 1 & 100 & N/A* & 83.3 & $\mathrm{~N}=2$ \\
\hline
\end{tabular}

Sensitivity, specificity and accuracy of diffusion tractography (DT) predictions for individual fiber tracts CST corticospinal tract, IFOF inferior fronto-occipital fasciculus, $O R$ optic radiations, ILF inferior longitudinal fasciculus, TP True Positive, TN True Negative, FP False Positive, FN False Negative, N/A Not available

*In the absence of True Negative instances, specificity cannot be meaningfully quantified

${ }^{a}$ Early deficit $=$ new or worse deficit emerging during surgery or in the immediate post-surgical period. Almost all deficits were transient, with the very small number (4\%) of persisting deficits each involving sensorimotor functions (CST), see "Results" section both techniques together. Finally, early post-operative DT offered added potential to anticipate recovery in patients with performance declines.

\section{Accuracy of pre-operative DT}

Our first study aim was to determine the sensitivity, specificity and accuracy of DTI in our sample of patients operated awake with subcortical stimulation. While numerous studies report using pre-operative DT for neuronavigation, few have quantified its sensitivity. Bello and colleagues [21] reported high sensitivity (88.6-98.2\%) and specificity (100\%) of DT predictions for motor and language pathways when assessed against intra-operative stimulation. Zhu et al. [29] obtained similar values ( $92.6 \%$ sensitivity, $93.2 \%$ specificity) for CST DT. We recorded similar sensitivity $(84.2-100 \%)$ and specificity (85.7-100\%) for 5 established surgically-relevant fiber tracts. We used more comprehensive criteria to evaluate DT, since 'gold standard' intra-operative subcortical stimulation varies in reliability and post-operative deficits can still arise 


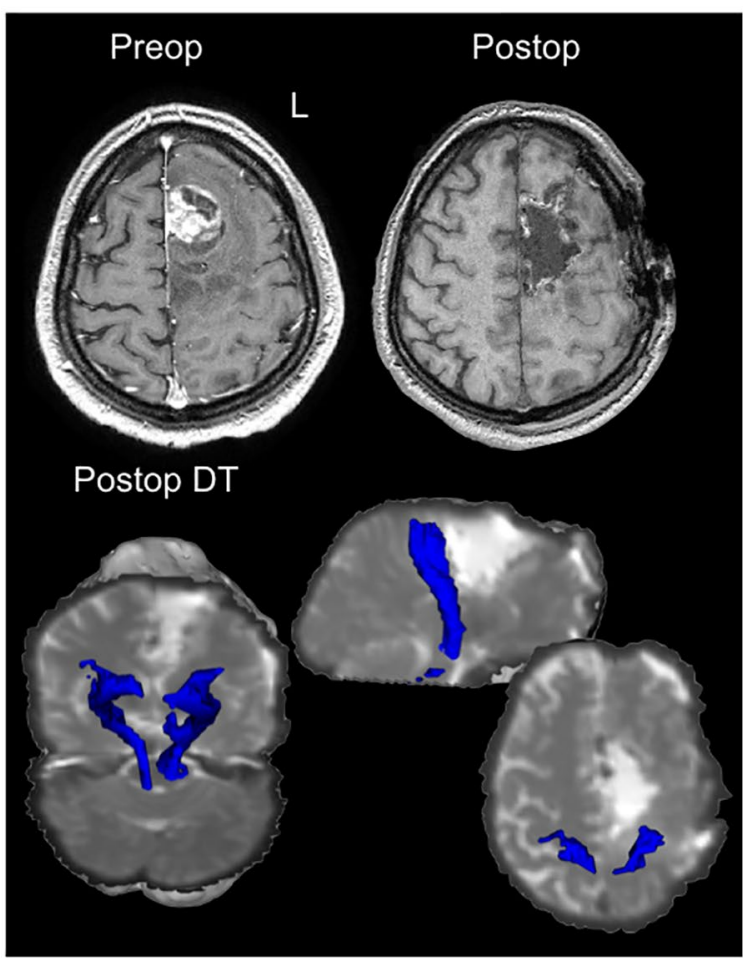

Fig. 3 Post-operative diffusion tractography to inform recovery potential in two patients with post-operative deficits. Left panel: A 42-year-old right handed male developed speech and motor deficits during resection of a radiologically transforming glioma in the medial frontal lobe. Speech recovered quickly over the first 3 days following surgery. Right hand function was absent immediately following surgery (0/5, MRC scale) and right leg function was significantly impaired (2/5). Post-operative diffusion tractography (DT) acquired $24 \mathrm{~h}$ after surgery showed preserved corticospinal (CST) fibers, supporting the diagnosis of a supplementary motor syndrome likely to recover. Hand function gradually returned on the 6th post-operative

[22]. When resecting tumor near eloquent tracts, some performance declines occurred without consistent stimulation findings (12 tracts, 9\%). Behavioral changes offer important additional validation of DT, since their presence indicates proximity to eloquent tracts DT aims to help preserve. We analyzed a large sample of patients operated awake, offering a way to directly observe patient behavior to assess tract proximity. However, many glioma surgeries are performed under general anesthesia. Motor/sensory evoked potentials offer an alternative method to minimize deficits when operating near sensory pathways with the patient awake or asleep [30].

\section{When results diverged}

Our second aim was to identify factors contributing to discrepancies between pre-operative DT and intra-operative stimulation. These included:

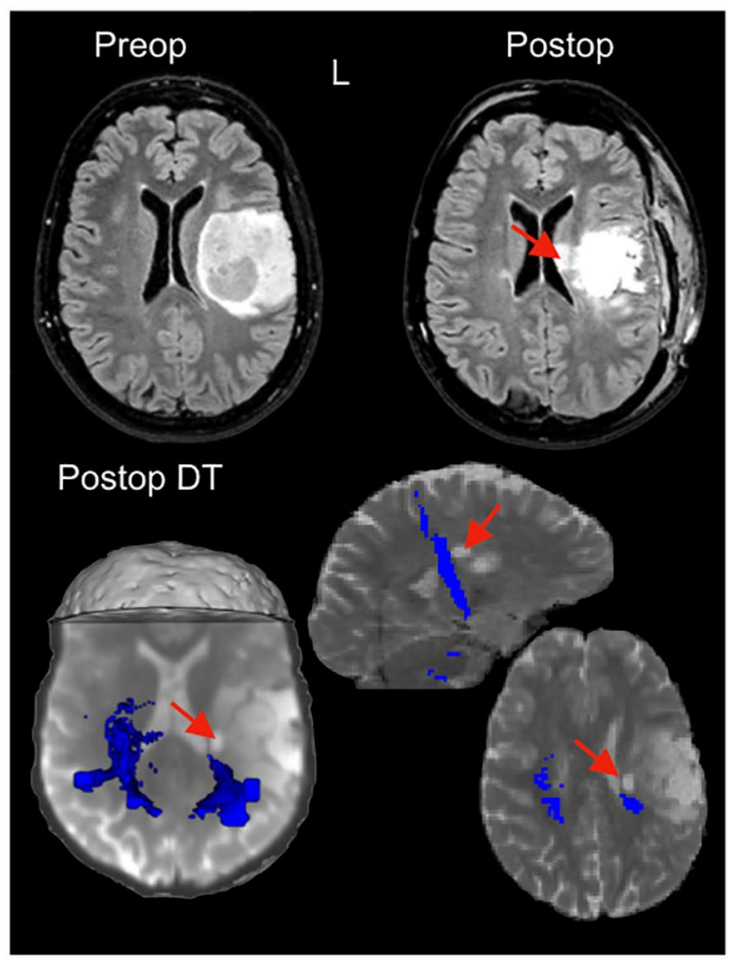

day; leg strength returned to normal by 1 month. Right panel: A 34-year-old right handed female developed sudden limb weakness. Immediate post-operative MRI identified an area of infarct affecting the corona radiata and body of the caudate nucleus. Post-operative DT demonstrated an asymmetrical CST, with fewer detected tracts adjacent to the infarct. Residual connections reaching the primary motor cortex, however, suggested some recovery potential. Hand function improved over the first 10 days after surgery, with additional gains in lower limb function with therapy which continued for 3 months post-surgery. However, she was still occasionally using a stick to walk

\section{Stimulation identifying tracts not seen with DT}

DT failures were rare, affecting $1 / 143$ tracts $(<1 \%)$. Insula gliomas can destroy the IFOF, and absence of IFOF in DT is predictive of achieving $>80 \%$ resection [17]. Conversely, in our case, DT did not identify one eloquent IFOF. This case highlights known challenges for DT when pathological signal (e.g. infiltrating tumor/edema) affects critical white matter regions, such as the temporo-parietal junction where numerous tracts intersect [31]. Diffusion acquisition sequences with high spatial $(\leq 2 \mathrm{~mm}$ isotropic voxels) and angular ( $\geq 60$ gradient directions) resolution, analyzed using sophisticated research models, improves DT in these regions [32]. Nonetheless, the potential for failures when diffusivity is disrupted re-affirms the need for confirmatory intra-operative monitoring whenever possible. 


\section{Stimulation not identifying DT-predicted tracts}

Three main factors explained why subcortical stimulation did not elicit behavioral change for 22 DT-predicted tracts:

1. Brain shift has been extensively described and affects neuronavigation based on pre-operative imaging. Excessive brain shift affected $2(1 \%)$ of our tract evaluations. Intraoperative MRI [33] or more accessible navigated ultrasound offer potential for real-time DT 'correction', but await further validation.

2. Residual tumor surrounded 11 DT-predicted tracts (8\%). In these cases, stimulation likely did not reach the targeted tract.

3. Functional variability. The arcuate, IFOF and CST generally produce consistent behavioral changes. Others, such as the ILF [34], appear functionally heterogeneous. Some studies omitted specificity analyses for functionally variable tracts [21]. Patients may also show atypical functional organization due to left-handedness, slowgrowing pathology or previous surgery (reviewed in [35]). Absence of stimulation-induced errors in these scenarios does not reflect inaccuracies of DT, but instead emphasizes differences in each tool's purpose. DT predicts the location of tracts, making no assumption about function, while stimulation evaluates tissue functionality without knowledge of tract locations. Which tract was stimulated is inferred from a patient's response [9]. However, some results are ambiguous (e.g. several tracts, when stimulated, induce anomia) or inconsistent (based on stimulation angle and current spread). 12 tracts were identified based on characteristic symptoms that could not be repeatedly, non-consecutively localized with stimulation, reflecting susceptibility to small variations in application. DT and stimulation are, therefore, highly complementary. Evaluation of one against the other neglects valuable independent information each technique provides.

Only four stimulation-silent DT-predicted tracts remained unexplained. In one left temporal glioblastoma patient, stimulation did not locate the ILF or optic radiations. Since the predicted IFOF, situated lateral to the optic radiations, was confirmed, optic fibers were likely not stimulated. Additionally, no visual symptoms were elicited through stimulation in a patient with a right medial parietal glioblastoma and near total resection. We did not conduct post-operative ophthalmological assessment and therefore cannot rule out subtle visual field deficits. Finally, the DTpredicted CST was not encountered in one patient with a left parietal glioblastoma who had gross total resection. Pre-operative DT indicated peri-tumoral edema displacing the CST. Due to an intra-operative cardiac event, resection stopped once visible tumor had been removed without attempting to reach functional boundaries. Classification of all four tracts as FP is therefore conservative, since resection likely did not reach at least two (excluding which, specificity becomes $81.8 \%$ ).

\section{Contributions to glioma surgery}

Benefits of pre-operative DT in planning and performing glioma resections were reviewed recently [36, 37]. DT is in our experience most helpful for a patient-centric approach and intra-operative guidance. Awake craniotomy is offered when an intrinsic tumor is located adjacent to presumed eloquent tissue, since striking a functional-oncological balance is vital [38]. Patients have a better understanding of surgical risks, a key part of the consent process, when the relationship between their tumor and surrounding fibers is discussed with them. Secondly, anticipating through DT the location of tracts helps the surgeon both to plan the surgical approach $[12,17,39]$ and where to target subcortical stimulation [21], especially when the length of surgery may not permit continued stimulation, or in areas (e.g. temporal fossa floor/falx) where patient discomfort can arise. Finally, DT-neuronavigation offers the surgeon confidence in proceeding with conservative resection when clinical factors preclude awake mapping, as in $3(3 \%)$ of our patients. At least for sensory pathways, DT can also complement neurophysiological monitoring techniques (e.g. motor and/or sensory evoked potentials) that are well-established to help achieve maximal resections and reduce new motor deficits when operating on patients either awake or asleep [40-42]. Additional DT applications include minimizing surgical morbidity and predicting extent of resection. A randomized controlled trial reported fewer performance deteriorations after surgeries informed by DT than performed without DT [26]. Concordantly, a recent prospective study reported positive predictive value of DT for resection extent [43], confirming previous findings [17].

The final aim of our study was to explore the potential for post-operative DT to predict functional recovery. Recovery from surgical white matter damage is thought to be limited [8]. While post-operative complications and brain swelling contribute to transient deterioration after surgery, the main concern is to rule out damage to eloquent tracts producing lasting impairment. Very few (4\%) of our patients experienced prolonged deficits. Nonetheless, post-operative DT distinguished a patient with a preserved CST who made a full recovery from another patient with a small infarct who required lengthy rehabilitation. Post-operative DT has rarely been reported, but another study demonstrated that postoperative language tract DT informed speech outcomes [24]. Together, these data suggest postoperative DT holds promise for predicting recovery potential. 


\section{Limitations}

Different tractography techniques produce varied results [44]. Deterministic algorithms have well-known limitations [45]. Advanced reconstruction techniques generally out-perform deterministic methods [46-49], but are not clinically licensed. Our deterministic-based results therefore under-estimate DT's potential. Additionally, some behavioral changes result from fluctuating patient co-operation, rather than proximity to eloquent tracts. Subjectivity was present in classifying intra-operative behavioral fluctuations, but minimized through consistent evaluation by the same assessor and joint decision-making with the operating surgeon. Likewise, there is not currently any definitive method of confirming that a particular (non-sensory) behavior was absolutely the result of stimulating a given white matter pathway. Within the limits of what is known of the function and organization of white matter, our interpretations of which tracts were associated with a given stimulation error/ behavioral change were guided by current practices [9] and recently proposed standardized testing protocols [27]. Finally, this was a retrospective study. Visibility of tractography results during navigation may bias the surgeon's interpretation. Here, tractography's accuracy was not recorded at the time of surgery, but analyzed later retrospectively and independently of the surgeon. Nonetheless, prospective randomized controlled studies are needed and underway in the UK ("FUTURE-GB", NIHR 127930) to evaluate DT's value in maximizing performance outcomes.

Supplementary Information The online version contains supplementary material available at https://doi.org/10.1007/s11060-021-03795-7.

Author contributions All authors contributed to the study conception and design. Material preparation, data collection and analysis were performed by NV, PP, VA, RS and MB. The first draft of the manuscript was written by NV and all authors commented on previous versions of the manuscript. All authors read and approved the final manuscript.

Funding No dedicated funding was raised for this study.

\section{Declarations}

Conflict of interest The authors report no competing interests.

Ethical approval The UK Health Research Authority approved this study (Ref: 19/HRA/2209).

Informed consent All patients gave written informed consent to surgery, including processing of their data for research.

Open Access This article is licensed under a Creative Commons Attribution 4.0 International License, which permits use, sharing, adaptation, distribution and reproduction in any medium or format, as long as you give appropriate credit to the original author(s) and the source, provide a link to the Creative Commons licence, and indicate if changes were made. The images or other third party material in this article are included in the article's Creative Commons licence, unless indicated otherwise in a credit line to the material. If material is not included in the article's Creative Commons licence and your intended use is not permitted by statutory regulation or exceeds the permitted use, you will need to obtain permission directly from the copyright holder. To view a copy of this licence, visit http://creativecommons.org/licenses/by/4.0/.

\section{References}

1. Jenkinson MD, Barone DG, Bryant A, Vale L, Bulbeck H, Lawrie TA, Hart MG, Watts C (2018) Intraoperative imaging technology to maximise extent of resection for glioma. Cochrane Database Syst Rev 1:CD012788. https://doi.org/10.1002/14651858.CD012 788.pub2

2. Wieshmann UC, Symms MR, Parker GJ, Clark CA, Lemieux L, Barker GJ, Shorvon SD (2000) Diffusion tensor imaging demonstrates deviation of fibres in normal appearing white matter adjacent to a brain tumour. J Neurol Neurosurg Psychiatry 68:501-503

3. Mori S, Frederiksen K, van Zijl PC, Stieltjes B, Kraut MA, Solaiyappan M, Pomper MG (2002) Brain white matter anatomy of tumor patients evaluated with diffusion tensor imaging. Ann Neurol 51:377-380

4. Capelle L, Fontaine D, Mandonnet E, Taillandier L, Golmard JL, Bauchet L, Pallud J, Peruzzi P, Baron MH, Kujas M, Guyotat J, Guillevin R, Frenay M, Taillibert S, Colin P, Rigau V, Vandenbos F, Pinelli C, Duffau H (2013) Spontaneous and therapeutic prognostic factors in adult hemispheric World Health Organization Grade II gliomas: a series of 1097 cases: clinical article. J Neurosurg 118:1157-1168. https://doi.org/10.3171/2013.1.JNS121

5. Xia L, Fang C, Chen G, Sun C (2018) Relationship between the extent of resection and the survival of patients with low-grade gliomas: a systematic review and meta-analysis. BMC Cancer 18:48. https://doi.org/10.1186/s12885-017-3909-x

6. Brown TJ, Brennan MC, Li M, Church EW, Brandmeir NJ, Rakszawski KL, Patel AS, Rizk EB, Suki D, Sawaya R, Glantz M (2016) Association of the extent of resection with survival in glioblastoma: a systematic review and meta-analysis. JAMA Oncol 2:1460-1469. https://doi.org/10.1001/jamaoncol.2016.1373

7. Duffau H, Capelle L (2004) Preferential brain locations of lowgrade gliomas. Cancer 100:2622-2626. https://doi.org/10.1002/ cncr.20297

8. Herbet G, Maheu M, Costi E, Lafargue G, Duffau H (2016) Mapping neuroplastic potential in brain-damaged patients. Brain $\mathbf{J}$ Neurol 139:829-844. https://doi.org/10.1093/brain/awv394

9. Duffau H (2015) Stimulation mapping of white matter tracts to study brain functional connectivity. Nat Rev Neurol 11:255-265. https://doi.org/10.1038/nrneurol.2015.51

10. Le Bihan D (2003) Looking into the functional architecture of the brain with diffusion MRI. Nat Rev Neurosci 4:469-480. https:// doi.org/10.1038/nrn1119

11. Fernandez-Miranda JC, Rhoton AL Jr., Alvarez-Linera J, Kakizawa Y, Choi C, de Oliveira EP (2008) Three-dimensional microsurgical and tractographic anatomy of the white matter of the human brain. Neurosurgery 62:989-1026. https://doi.org/10.1227/ 01.neu.0000333767.05328.49

12. Bello L, Castellano A, Fava E, Casaceli G, Riva M, Scotti G, Gaini SM, Falini A (2010) Intraoperative use of diffusion tensor imaging fiber tractography and subcortical mapping for resection of gliomas: technical considerations. Neurosurg Focus 28:E6. https:// doi.org/10.3171/2009.12.FOCUS09240

13. Celtikci P, Fernandes-Cabral DT, Yeh FC, Panesar SS, Fernandez-Miranda JC (2018) Generalized q-sampling imaging fiber 
tractography reveals displacement and infiltration of fiber tracts in low-grade gliomas. Neuroradiology 60:267-280. https://doi. org/10.1007/s00234-018-1985-5

14. Voets NL, Bartsch A, Plaha P (2017) Brain white matter fibre tracts: a review of functional neuro-oncological relevance. J Neurol Neurosurg Psychiatry. https://doi.org/10.1136/ jnnp-2017-316170

15. Castellano A, Bello L, Michelozzi C, Gallucci M, Fava E, Iadanza A, Riva M, Casaceli G, Falini A (2012) Role of diffusion tensor magnetic resonance tractography in predicting the extent of resection in glioma surgery. Neuro Oncol 14:192-202. https://doi.org/ 10.1093/neuonc/nor188

16. Ius T, Isola M, Budai R, Pauletto G, Tomasino B, Fadiga L, Skrap M (2012) Low-grade glioma surgery in eloquent areas: volumetric analysis of extent of resection and its impact on overall survival. A single-institution experience in 190 patients: clinical article. J Neurosurg 117:1039-1052. https://doi.org/10.3171/2012.8.JNS12 393

17. Martino J, Mato D, de Lucas EM, Garcia-Porrero JA, Gabarros A, Fernandez-Coello A, Vazquez-Barquero A (2015) Subcortical anatomy as an anatomical and functional landmark in insulo-opercular gliomas: implications for surgical approach to the insular region. J Neurosurg 123:1081-1092. https://doi.org/10.3171/2014. 11.JNS141992

18. Berman JI, Berger MS, Chung SW, Nagarajan SS, Henry RG (2007) Accuracy of diffusion tensor magnetic resonance imaging tractography assessed using intraoperative subcortical stimulation mapping and magnetic source imaging. J Neurosurg 107:488-494. https://doi.org/10.3171/JNS-07/09/0488

19. Nimsky C, Ganslandt O, Hastreiter P, Wang R, Benner T, Sorensen AG, Fahlbusch R (2007) Preoperative and intraoperative diffusion tensor imaging-based fiber tracking in glioma surgery. Neurosurgery 61:178-185. https://doi.org/10.1227/01.neu.00002 $79214.00139 .3 b$

20. Mikuni N, Okada T, Enatsu R, Miki Y, Hanakawa T, Urayama S, Kikuta K, Takahashi JA, Nozaki K, Fukuyama H, Hashimoto N (2007) Clinical impact of integrated functional neuronavigation and subcortical electrical stimulation to preserve motor function during resection of brain tumors. J Neurosurg 106:593-598. https://doi.org/10.3171/jns.2007.106.4.593

21. Bello L, Gambini A, Castellano A, Carrabba G, Acerbi F, Fava E, Giussani C, Cadioli M, Blasi V, Casarotti A, Papagno C, Gupta AK, Gaini S, Scotti G, Falini A (2008) Motor and language DTI Fiber Tracking combined with intraoperative subcortical mapping for surgical removal of gliomas. Neuroimage 39:369-382. https:// doi.org/10.1016/j.neuroimage.2007.08.031

22. Shinoura N, Midorikawa A, Yamada R, Hana T, Saito A, Hiromitsu K, Itoi C, Saito S, Yagi K (2013) Awake craniotomy for brain lesions within and near the primary motor area: a retrospective analysis of factors associated with worsened paresis in 102 consecutive patients. Surg Neurol Int 4:149. https://doi.org/10. 4103/2152-7806.122003

23. Mikuni N, Okada T, Enatsu R, Miki Y, Urayama S, Takahashi JA, Nozaki K, Fukuyama H, Hashimoto N (2007) Clinical significance of preoperative fibre-tracking to preserve the affected pyramidal tracts during resection of brain tumours in patients with preoperative motor weakness. J Neurol Neurosurg Psychiatry 78:716-721. https://doi.org/10.1136/jnnp.2006.099952

24. Caverzasi E, Hervey-Jumper SL, Jordan KM, Lobach IV, Li J, Panara V, Racine CA, Sankaranarayanan V, Amirbekian B, Papinutto N, Berger MS, Henry RG (2015) Identifying preoperative language tracts and predicting postoperative functional recovery using HARDI q-ball fiber tractography in patients with gliomas. J Neurosurg. https://doi.org/10.3171/2015.6.JNS142203

25. Oda K, Yamaguchi F, Enomoto H, Higuchi T, Morita A (2018) Prediction of recovery from supplementary motor area syndrome after brain tumor surgery: preoperative diffusion tensor tractography analysis and postoperative neurological clinical course. Neurosurg Focus 44:E3. https://doi.org/10.3171/2017.12.FOCUS 17564

26. Wu JS, Zhou LF, Tang WJ, Mao Y, Hu J, Song YY, Hong XN, Du GH (2007) Clinical evaluation and follow-up outcome of diffusion tensor imaging-based functional neuronavigation: a prospective, controlled study in patients with gliomas involving pyramidal tracts. Neurosurgery 61:935-948. https://doi.org/10.1227/01.neu. 0000303189.80049.ab

27. Mandonnet E, Sarubbo S, Duffau H (2017) Proposal of an optimized strategy for intraoperative testing of speech and language during awake mapping. Neurosurg Rev 40:29-35. https://doi.org/ 10.1007/s10143-016-0723-x

28. Rofes A, Mandonnet E, Godden J, Baron MH, Colle H, Darlix A, de Aguiar V, Duffau H, Herbet G, Klein M, Lubrano V, Martino J, Mathew R, Miceli G, Moritz-Gasser S, Pallud J, Papagno C, Rech F, Robert E, Rutten GJ, Santarius T, Satoer D, Sierpowska J, Smits A, Skrap M, Spena G, Visch E, De Witte E, Zetterling M, Wager M (2017) Survey on current cognitive practices within the European Low-Grade Glioma Network: towards a European assessment protocol. Acta Neurochir 159:1167-1178. https://doi. org/10.1007/s00701-017-3192-2

29. Zhu FP, Wu JS, Song YY, Yao CJ, Zhuang DX, Xu G, Tang WJ, Qin ZY, Mao Y, Zhou LF (2012) Clinical application of motor pathway mapping using diffusion tensor imaging tractography and intraoperative direct subcortical stimulation in cerebral glioma surgery: a prospective cohort study. Neurosurgery 71:1170-1183. https://doi.org/10.1227/NEU.0b013e318271bc61

30. Ottenhausen M, Krieg SM, Meyer B, Ringel F (2015) Functional preoperative and intraoperative mapping and monitoring: increasing safety and efficacy in glioma surgery. Neurosurg Focus 38:E3. https://doi.org/10.3171/2014.10.FOCUS14611

31. Martino J, da Silva-Freitas R, Caballero H, Marco de Lucas E, Garcia-Porrero JA, Vazquez-Barquero A (2013) Fiber dissection and diffusion tensor imaging tractography study of the temporoparietal fiber intersection area. Neurosurgery 72:87-97. https:// doi.org/10.1227/NEU.0b013e318274294b

32. Vos SB, Aksoy M, Han Z, Holdsworth SJ, Maclaren J, Viergever MA, Leemans A, Bammer R (2016) Trade-off between angular and spatial resolutions in in vivo fiber tractography. Neuroimage 129:117-132. https://doi.org/10.1016/j.neuroimage.2016.01.011

33. Nimsky C, Ganslandt O, Hastreiter P, Wang R, Benner T, Sorensen AG, Fahlbusch R (2005) Preoperative and intraoperative diffusion tensor imaging-based fiber tracking in glioma surgery. Neurosurgery 56:130-137

34. Mandonnet E, Nouet A, Gatignol P, Capelle L, Duffau H (2007) Does the left inferior longitudinal fasciculus play a role in language? A brain stimulation study. Brain J Neurol 130:623-629. https://doi.org/10.1093/brain/awl361

35. Cargnelutti E, Ius T, Skrap M, Tomasino B (2020) What do we know about pre- and postoperative plasticity in patients with glioma? A review of neuroimaging and intraoperative mapping studies. NeuroImage Clin 28:102435. https://doi.org/10.1016/j. nicl.2020.102435

36. Essayed WI, Zhang F, Unadkat P, Cosgrove GR, Golby AJ, O'Donnell LJ (2017) White matter tractography for neurosurgical planning: a topography-based review of the current state of the art. NeuroImage Clin 15:659-672. https://doi.org/10.1016/j.nicl. 2017.06.011

37. Vanderweyen DC, Theaud G, Sidhu J, Rheault F, Sarubbo S, Descoteaux M, Fortin D (2020) The role of diffusion tractography in refining glial tumor resection. Brain Struct Funct 225:14131436. https://doi.org/10.1007/s00429-020-02056-Z

38. Duffau H, Mandonnet E (2013) The "onco-functional balance" in surgery for diffuse low-grade glioma: integrating the extent 
of resection with quality of life. Acta Neurochir 155:951-957. https://doi.org/10.1007/s00701-013-1653-9

39. Frati A, Pesce A, D'Andrea G, Fraschetti F, Salvati M, Cimatti M, Esposito V, Raco A (2018) A purely functional Imaging based approach for transcortical resection of lesion involving the dominant atrium: towards safer, imaging-guided, tailored cortico-leucotomies. J Clin Neurosci Off J Neurosurg Soc Australas 50:252-261. https://doi.org/10.1016/j.jocn.2018.01.045

40. Keles GE, Lundin DA, Lamborn KR, Chang EF, Ojemann G, Berger MS (2004) Intraoperative subcortical stimulation mapping for hemispherical perirolandic gliomas located within or adjacent to the descending motor pathways: evaluation of morbidity and assessment of functional outcome in 294 patients. J Neurosurg 100:369-375. https://doi.org/10.3171/jns.2004.100.3.0369

41. Bello L, Riva M, Fava E, Ferpozzi V, Castellano A, Raneri F, Pessina F, Bizzi A, Falini A, Cerri G (2014) Tailoring neurophysiological strategies with clinical context enhances resection and safety and expands indications in gliomas involving motor pathways. Neuro Oncol 16:1110-1128. https://doi.org/10.1093/ neuonc/not327

42. Shiban E, Krieg SM, Haller B, Buchmann N, Obermueller T, Boeckh-Behrens T, Wostrack M, Meyer B, Ringel F (2015) Intraoperative subcortical motor evoked potential stimulation: how close is the corticospinal tract? J Neurosurg 123:711-720. https:// doi.org/10.3171/2014.10.JNS141289

43. Aibar-Duran JA, de Quintana-Schmidt C, Alvarez Holzpafel MJ, Hernandez FM, Cortes CA, Martinez GV, Bertran GC (2020) Intraoperative use and benefits of tractography in awake surgery patients. World Neurosurg 137:e347-e353. https://doi.org/10. 1016/j.wneu.2020.01.210

44. Pujol S, Wells W, Pierpaoli C, Brun C, Gee J, Cheng G, Vemuri B, Commowick O, Prima S, Stamm A, Goubran M, Khan A, Peters T, Neher P, Maier-Hein KH, Shi Y, Tristan-Vega A, Veni G, Whitaker R, Styner M, Westin CF, Gouttard S, Norton I, Chauvin L,
Mamata H, Gerig G, Nabavi A, Golby A, Kikinis R (2015) The DTI challenge: toward standardized evaluation of diffusion tensor imaging tractography for neurosurgery. J Neuroimaging Off J Am Soc Neuroimaging 25:875-882. https://doi.org/10.1111/jon.12283

45. Catani M (2007) From hodology to function. Brain J Neurol 130:602-605. https://doi.org/10.1093/brain/awm008

46. Li Z, Peck KK, Brennan NP, Jenabi M, Hsu M, Zhang Z, Holodny AI, Young RJ (2013) Diffusion tensor tractography of the arcuate fasciculus in patients with brain tumors: comparison between deterministic and probabilistic models. J Biomed Sci Eng 6:192200. https://doi.org/10.4236/jbise.2013.62023

47. Mandelli ML, Berger MS, Bucci M, Berman JI, Amirbekian B, Henry RG (2014) Quantifying accuracy and precision of diffusion MR tractography of the corticospinal tract in brain tumors. J Neurosurg 121:349-358. https://doi.org/10.3171/2014.4.JNS13 1160

48. Jenabi M, Peck KK, Young RJ, Brennan N, Holodny AI (2015) Identification of the corticobulbar tracts of the tongue and face using deterministic and probabilistic DTI fiber tracking in patients with brain tumor. AJNR Am J Neuroradiol 36:2036-2041. https:// doi.org/10.3174/ajnr.A4430

49. Munnich T, Klein J, Hattingen E, Noack A, Herrmann E, Seifert V, Senft C, Forster MT (2019) Tractography verified by intraoperative magnetic resonance imaging and subcortical stimulation during tumor resection near the corticospinal tract. Oper Neurosurg (Hagerstown) 16:197-210. https://doi.org/10.1093/ons/ opy062

Publisher's Note Springer Nature remains neutral with regard to jurisdictional claims in published maps and institutional affiliations. 